\title{
Extended Tersoff potential for boron nitride: Energetics and elastic properties of pristine and defective $h$-BN
}

\author{
J. H. Los, ${ }^{1,}$ J. M. H. Kroes, ${ }^{1}$ K. Albe, ${ }^{2}$ R. M. Gordillo, ${ }^{3}$ M. I. Katsnelson, ${ }^{1}$ and A. Fasolino ${ }^{1}$ \\ ${ }^{1}$ Radboud University, Institute for Molecules and Materials, Heyendaalseweg 135, 6525AJ Nijmegen, The Netherlands \\ ${ }^{2}$ Technische Universität Darmstadt, Institut für Materialwissenschaft, Fachgebiet Materialmodellierung, \\ Jovanka-Bontschits-Str. 2, D-64287 Darmstadt, Germany \\ ${ }^{3}$ Aix-Marseille Université and CNRS, CINAM UMR 7325, 13288 Marseille, France
}

(Received 24 May 2017; revised manuscript received 23 October 2017; published 20 November 2017)

\begin{abstract}
We present an extended Tersoff potential for boron nitride (BN-ExTeP) for application in large scale atomistic simulations. BN-ExTeP accurately describes the main low energy $\mathrm{B}, \mathrm{N}$, and $\mathrm{BN}$ structures and yields quantitatively correct trends in the bonding as a function of coordination. The proposed extension of the bond order, added to improve the dependence of bonding on the chemical environment, leads to an accurate description of point defects in hexagonal $\mathrm{BN}(h$-BN) and cubic $\mathrm{BN}(c$-BN). We have implemented this potential in the molecular dynamics LAMMPS code and used it to determine some basic properties of pristine $2 \mathrm{D} h$-BN and the elastic properties of defective $h$-BN as a function of defect density at zero temperature. Our results show that there is a strong correlation between the size of the static corrugation induced by the defects and the weakening of the in-plane elastic moduli.
\end{abstract}

DOI: 10.1103/PhysRevB.96.184108

\section{INTRODUCTION}

Following the discovery of graphene, many other 2D materials have been identified [1]. Of these, monolayer hexagonal boron nitride $(h-\mathrm{BN})$ is attracting increasing attention as a prototype substrate for graphene thanks to its similar structure, chemical inertness, and the insulating character [2,3]. The large 5-6 eV band gap [4] makes $h$-BN transparent, whence its nickname "white graphene," and makes it suitable for application in deep UV optoelectronic devices [5,6].

Besides $h$-BN, BN and the pure components B and N exist in many competing phases. Different classical potentials have been parametrized for B-N systems, including a number of Tersoff potentials [7-10]. For $h$-BN, Kinaci et al. parametrized a Tersoff potential to describe phonons and thermal transport $[9,10]$ while Matsunaga et al. parametrized a Tersoff potential to study cubic boron carbonitrides [8]. Also several ReaxFF potentials have been parametrized to describe liquids with molecules including boron and nitrogen [11,12]. Of the existing potentials for $\mathrm{BN}$ proposed thus far, however, none are meant to describe the larger class of solid phases of $\mathrm{B}, \mathrm{N}$, and $\mathrm{BN}$, as well as various defects that typically occur in CVD grown and irradiated materials [13-15].

Here we introduce BN-ExTeP, an extended Tersoff potential for BN specifically aimed at describing correctly the dependence on coordination and chemical environment of the bonding occurring in $\mathrm{B}, \mathrm{N}$, and $\mathrm{B}-\mathrm{N}$ systems. The normal Tersoff part of the potential improves an earlier Tersoff potential by Albe and Möller for BN [7,16]. For the here presented potential, special attention is paid to achieving an optimal description of 2D $h$-BN, including its thermal stability, lattice parameter, and elastic properties. Regarding its elastic properties, it solves the artifact of the earlier potential $[7,16]$ that yields a too large uniaxial modulus and

\footnotetext{
*j.los@science.ru.nl
}

a negative Poisson's ratio, in disagreement with $a b$ initio calculations $[17,18]$. In addition, BN-ExTeP yields a lattice parameter for $h$-BN in perfect agreement with the experimental value, which makes it, in conjunction with a potential for carbon, particularly suitable for application to graphene/ $h$-BN heterostructures requiring an accurate description of the lattice mismatch between graphene and $h$-BN. To improve the description of nonpristine structures, in particular defected $h$-BN and $c-\mathrm{BN}$, a correction to the bond order is introduced that explicitly takes into account the chemical environments of the atoms involved in the bond, as suggested in Ref. [16]. While the pure B parametrization in BN-ExTeP is practically the same as in Ref. [16], the parametrization of the pure $\mathrm{N}$ part has been modified to improve the binding energies of the relatively stable pure $\mathrm{N} 2 \mathrm{D}$ honeycomb and diamond lattices and the $\mathrm{BN}$ interactions have been reparametrized to improve the description of $h$-BN and $c-\mathrm{BN}$.

The complete BN-ExTeP has been implemented in LAMMPS [19] and allows for large scale molecular dynamics simulations of BN systems. Here, we use it to study the elastic properties of defective $h-\mathrm{BN}$ at $T=0 \mathrm{~K}$ as a function of defect density. For $h$-BN with randomly distributed defects we find a weakening of the elastic constants with increasing defect density that is much more pronounced for those defects that induce large static corrugations of the layer.

In Sec. II we give a brief description of the potential, for which we provide the numerical parametrization in Sec. III. In Sec. III A we present the parametrization of the Tersoff potential, optimized for the description of reference $\mathrm{B}, \mathrm{N}$, and $\mathrm{BN}$ molecular and crystal structures not involving the correction term. In Sec. III B, we then compare the structure and energetics of many point defects in $h$-BN and $c$-BN and of binary molecular structures to $a b$ initio results to complete the parametrization of the correction term. Our results from large scale simulations of pristine and defective $2 \mathrm{D} h$-BN will be presented in Secs. IV and V, respectively. Conclusions and perspectives are given in Sec. VI. 


\section{DESCRIPTION OF THE POTENTIAL}

BN-ExTeP extends the classical Tersoff potential [20] with a correction to the bond order that allows us in particular to discriminate bonding situations where the coordination numbers are identical but chemical identities are not. For completeness, the description below also includes the basic form of the Tersoff potential [20].

The total binding energy of the system is given by

$$
E=\frac{1}{2} \sum_{i, j} f_{C}\left(r_{i j}\right)\left[V_{R}\left(r_{i j}\right)-B_{i j} V_{A}\left(r_{i j}\right)\right] .
$$

The double sum here runs over all atoms $i$ and $j$, and $f_{C}$ is a smooth cutoff function:

$$
f_{C}(r)= \begin{cases}1 & r \leqslant R-D \\ \frac{1}{2}\left[1-\sin \left(\frac{\pi(r-R)}{2 D}\right)\right] & |R-r|<D . \\ 0 & r \geqslant R+D\end{cases}
$$

$V_{R}$ and $V_{A}$ are the repulsive and attractive potentials, respectively,

$$
\begin{aligned}
V_{R}(r) & =\frac{D_{0}}{S-1} \exp \left[-\beta \sqrt{2 S}\left(r-r_{0}\right)\right], \\
V_{A}(r) & =\frac{S D_{0}}{S-1} \exp \left[-\beta \sqrt{2 / S}\left(r-r_{0}\right)\right],
\end{aligned}
$$

so that the dimer bond energy, $V_{R}(r)-V_{A}(r)$, has a minimum at $r=r_{0}$ equal to $-D_{0} . B_{i j}$ is the total bond order,

$$
B_{i j}=\frac{1}{2}\left(b_{i j}+b_{j i}\right)+F_{i j},
$$

where $b_{i j}$ is the usual Tersoff bond order and $F_{i j}$ is the proposed correction.

The Tersoff bond order $b_{i j}$ is a many-body term involving the relative positions of nearest neighbors reading

$$
b_{i j}=\left(1+\gamma^{n} \chi_{i j}^{n}\right)^{-1 /(2 n)} \text {, }
$$

where

$$
\chi_{i j}=\sum_{k \neq i, j} f_{C}\left(r_{i k}\right) g\left(\theta_{i j k}\right) \exp \left(\lambda_{3}^{3}\left(r_{i j}-r_{i k}\right)^{3}\right),
$$

with $\theta_{i j k}$ the angle between $\mathbf{r}_{i j}$ and $\mathbf{r}_{i k}$ and

$$
g(\theta)=1+\frac{c^{2}}{d^{2}}-\frac{c^{2}}{d^{2}+(h-\cos \theta)^{2}} .
$$

The correction term reads

$$
F_{i j}=F_{\text {env }}\left(N_{i j}, N_{j i}\right) F_{I J}\left(N_{i j}^{\bar{I}}, N_{j i}^{\bar{J}}\right),
$$

where $F_{\text {env }}$ is an envelope function that smoothly switches off the correction term for the BN dimer and for high (total) coordinations $N_{i j}$ and/or $N_{j i}$, and where $F_{I J}\left(N_{i j}^{\bar{I}}, N_{j i}^{\bar{J}}\right)$ is the correction term proposed in Ref. [16], which depends on the partial, reduced coordination numbers $N_{i j}^{\bar{I}}$ and $N_{j i}^{\bar{J}}$ for neighbors with a chemical identity $\bar{I}$ and $\bar{J}$ other than the identity $I$ of atom $i$ and $J$ of atom $j$, respectively, with $I, J=\mathrm{B}, \mathrm{N}$. The total, reduced coordination $N_{i j}$, excluding neighbor $j$, is given by:

$$
N_{i j}=\sum_{k \neq i, j} f_{C}\left(r_{i k}\right),
$$

whereas the partial, reduced coordination $N_{i j}^{\bar{I}}$ is given by:

$$
N_{i j}^{\bar{I}}=\sum_{k \neq i, j} f_{C}\left(r_{i k}\right) \delta_{\bar{I}, K},
$$

where $\delta_{\bar{I}, K}$ is one if the chemical identity $K$ of atom $k$ is not equal to the chemical identity $I$ of atom $i$ and zero otherwise. The envelope function reads:

$$
F_{\text {env }}\left(N_{i j}, N_{j i}\right)=F_{\text {env }, 0}\left(N_{i j}, N_{j i}\right) F_{\text {env }, 4}\left(N_{i j}\right) F_{\text {env }, 4}\left(N_{j i}\right)
$$

with:

$$
F_{\text {env }, 0}(x, y)=1-(1-x)^{2}(1-y)^{2}
$$

for $x, y<1$ and $F_{\mathrm{env}, 0}(x, y)=1$ otherwise, and

$$
F_{\text {env, } 4}(x)=(2 x-5)(4-x)^{2}
$$

for $3<x<4$, while $F_{\text {env }, 4}(x)=1$ for $x \leqslant 3$ and $F_{\text {env }, 4}(x)=0$ for $x \geqslant 4$. This envelope guarantees continuity up to first order derivatives as required for molecular dynamics simulation.

The low energy structures in BN systems are low coordinated $(\leqslant 4)$ and accordingly the correction term parameters $F_{I J}$ are fitted to low coordination configurations. The envelope function in Eq. (9) is applied to avoid unphysical stabilization of configurations with high coordination $(>4)$ due to correction terms. Without $F_{\text {env }}$ we found unphysical, relatively high coordinated configurations appearing during melting of $c$-BN or $h$-BN.

For noninteger arguments, $F_{I J}$ is evaluated using a spline, identical to that used for the conjugation term in LCBOP [21], based on the values $F_{I J}$ and the first order partial derivatives of $F_{I J}$ at the integer, grid points $\left(N_{i j}^{\bar{I}}, N_{j i}^{\bar{J}}\right)$ for $0 \leqslant N_{i j}^{\bar{I}} \leqslant 4$ and $0 \leqslant N_{j i}^{\bar{J}} \leqslant 4$. The values of $F_{I J}$ for $N_{i j}^{\bar{I}}=4$ and/or $N_{j i}^{\bar{J}}=4$ are set equal to $F_{I J}\left(4, N_{j i}^{\bar{J}}\right)=F_{I J}\left(3, N_{j i}^{\bar{J}}\right)$ and $F_{I J}\left(N_{i j}^{\bar{I}}, 4\right)=F_{I J}\left(N_{i j}^{\bar{I}}, 3\right)$. Partial derivatives with respect to $N_{i j}^{\bar{I}}$ are set to zero at grid points with $N_{j i}^{\bar{J}}=0,3$ and 4 , and partial derivatives with respect to $N_{j i}^{\bar{J}}$ are set to zero at grid points with $N_{i j}^{\bar{I}}=0,3$, and 4 . In the other cases, the partial derivative at a given grid point is also set to zero if the values of $F_{i j}$ at the two neighboring grid points are both smaller or both larger than the value of $F_{i j}$ at the given grid point. In the remaining cases, the partial derivatives are computed by a centered, finite difference expression based on the values at these neighboring grid points, e.g., $\partial F_{i j} / \partial N_{i j}^{\bar{I}}=\left(F_{i j}\left(N_{i j}^{\bar{I}}+1, N_{j i}^{\bar{J}}\right)-F_{i j}\left(N_{i j}^{\bar{I}}-1, N_{j i}^{\bar{J}}\right)\right) / 2$ at the grid point $\left(N_{i j}^{\bar{I}}, N_{j i}^{\bar{J}}\right)$.

\section{PARAMETRIZATION}

\section{A. Tersoff potential}

We first parametrize the Tersoff part of BN-ExTeP by least square fitting to reference values for $\mathrm{B}, \mathrm{N}$, and $\mathrm{BN}$ structures with variable coordination environments. Most of the reference values were obtained from $a b$ initio calculations within density functional theory (DFT) using the DMol code and reported in Ref. [16]. Where available, these values were compared to experimental data or other ab initio values from the literature. For all defects in $h$-BN apart from the intercalation defects (see Tables V and VI), we also performed DFT calculations 
TABLE I. Tersoff potential parameters for BN systems. The parameters for BB were taken from Ref. [16], except for $\lambda_{3}$. For $\mathrm{NN}$, part the parameters were adopted from Refs. [7,16], but $n, \gamma$, $c, d$, and $h$ where refitted in this work. The BN part was completely refitted in the present work.

\begin{tabular}{llll}
\hline \hline & \multicolumn{1}{c}{$\mathrm{BB}$} & \multicolumn{1}{c}{$\mathrm{NN}$} & \multicolumn{1}{c}{$\mathrm{BN}$} \\
\hline$D_{0}(\mathrm{eV})$ & 3.08 & 9.91 & 6.36 \\
$r_{0}(\AA)$ & 1.59 & 1.11 & 1.3253868267 \\
$\beta\left(\AA^{-1}\right)$ & 1.84 & 1.92787 & 1.9931611199 \\
$S$ & 1.065264 & 1.0769 & 1.0952902519 \\
$n$ & 1.142247 & 0.665 & 0.6576543657 \\
$\gamma$ & 0.01498959 & 0.019251 & 0.0027024851 \\
$c$ & 26617.3 & 23.5 & 306.586555205 \\
$d$ & 141.2 & 3.75 & 10.0 \\
$h$ & -0.13 & -0.4 & -0.7218 \\
$\lambda_{3}\left(\AA^{-1}\right)$ & 0 & 0 & 0 \\
$R(\AA)$ & 2.0 & 2.0 & 2.0 \\
$D(\AA)$ & 0.2 & 0.2 & 0.2 \\
\hline \hline
\end{tabular}

using the SIESTA code [22]. These DFT calculations were done using the Perdew-Burke-Ernzerhof (PBE) parametrization of the generalized gradient approximation [23] for the exchange-correlation functional. We used norm-conserving Troullier-Martin pseudopotentials [24] and a numerical atomic orbital with double- $\zeta$ plus polarization basis set to represent the valence electrons with a real-space integration grid of $300 \mathrm{Ry}$. Periodic boundary conditions were applied to a supercell of $43.23 \times 44.94 \AA$, consisting of 720 atoms for pristine $h$-BN, and a $4 \times 4 k$-point sampling was used.

The parametrization of the Tersoff part of BN-ExTeP is given in Table I. The parameters for pure B, except for $\lambda_{3}$, and part for the parameters for pure $\mathrm{N}$ were taken from Refs. [16]. A summary of the properties of pure $\mathrm{B}$ and $\mathrm{N}$ reference structures and of the $\mathrm{BN}$ compound structures $h-\mathrm{BN}$ and $c-\mathrm{BN}$ is given in Tables II and III, respectively.

Table II reveals that, for B, the most stable low temperature structures are those with intermediate coordinations, like the icosahedral cluster with fivefold coordinated atoms and the related $\alpha$-boron crystal structure containing sixfold and sevenfold coordinated atoms. The latter structure is a polymorph made of a close packing of chemically interconnected icosahedra. The most stable crystalline phase of $\mathrm{B}$ at ambient conditions is, however, the $\beta$-polymorph, a much more complex structure which can be described as an icosahedral central unit, icosahedrally surrounded by other icosahedra, with a rhombohedral unit cell containing 105 atoms. Obviously, one cannot expect, nor is it the aim here, to describe such structures with high accuracy with a simple Tersoff potential. Therefore, for convenience only the $\alpha$ phase was used as a reference in the fitting procedure, which is enough for obtaining the correct quantitative trends in the coordination dependence of the binding energy per atom. We note that the $\alpha$ and $\beta$ phase have rather similar structures, both being made of icosahedral units, with the $\beta$ phase being more stable by only about $0.1 \mathrm{eV} /$ atom [25]. The underestimation of the lattice parameter of the FCC structure is due to the choice of the cutoff range applied, which is suitable for relatively stable structures but not for the FCC lattice.
TABLE II. Ground state equilibrium nearest neighbor distances $r_{\text {eq, } 0}$ and binding energies per atom $E_{b}$ for pure B (upper table) and $\mathrm{N}$ (lower table) reference structures according to BN-ExTeP, compared to references values (in parenthesis) mostly obtained from $a b$ initio calculations [16,26]. For $\alpha$-boron, experimental data are used $[28,29]$. Reference values in square brackets were not used in the fitting procedure.

\begin{tabular}{lcc}
\hline \hline Boron structures & $r_{\mathrm{eq}, 0}(\AA)$ & $E_{b}(\mathrm{eV} /$ atom $)$ \\
\hline dimer & $1.59(1.59)$ & $1.540(1.540)$ \\
$B_{3}$ (triangle) & $1.63(1.58)$ & $2.770(3.110)$ \\
$B_{3}$ (linear) & $1.62[1.52]$ & $1.900[2.486]$ \\
$B_{4}$ (rhombus) & $1.64[1.54]$ & $3.250[3.723]$ \\
$B_{12}$ (icosahedron) & $1.74(1.72)$ & $5.125(5.250)$ \\
$\alpha$-boron & $1.80 \pm 0.03$ & $5.650(5.810)$ \\
& $(1.77 \pm 0.2)$ & \\
FCC & $1.94(1.21)$ & $5.332(5.700)$ \\
Nitrogen structures & & \\
dimer & $r_{\mathrm{eq}, 0}(\AA)$ & $E_{b}(\mathrm{eV} / \mathrm{atom})$ \\
$N_{3}$ (linear) & $1.110(1.11)$ & $4.955(4.955)$ \\
$N_{3}$ (triangle) & $1.307(1.21)$ & $3.788(3.653)$ \\
honeycomb hexagonal & 1.645 & 2.179 \\
diamond & $1.521(1.51)$ & $4.647(4.560)$ \\
simple cubic & $1.635(1.79)$ & $4.494(4.240)$ \\
FCC & $1.943(2.00)$ & $2.447(3.480)$ \\
\hline \hline
\end{tabular}

Unlike B, N prefers low coordination with the dimer as the energetically most stable structure, as correctly reproduced by the Tersoff potential. For N, also the energies of the low coordinated honeycomb and diamond lattices are quite well described, while the higher coordinated simple cubic and

TABLE III. Overview of ground state properties of $h$-BN and $c$-BN, compared to those from $a b$ initio calculations and experimental data. Binding energies $E_{b}$ are given in $\mathrm{eV}$ per BN chemical unit (c.u.). The $2 \mathrm{D}$ shear modulus $\mu_{2 D}$, Young's modulus $E_{2 D}$, and Poisson's ratio $\nu_{2 D}$ were determined from $B_{2 D}$ and $C_{11,2 D}$ using Eq. (15). Elastic constants were calculated including relaxation, except for $C_{44}^{0}$, the adiabatic shear elastic constant for $c$-BN

\begin{tabular}{llll}
\hline \hline $\boldsymbol{h}$-BN & BN-ExTeP & \multicolumn{1}{c}{ ab initio } & \multicolumn{1}{c}{ Experiment } \\
\hline$a(\AA)$ & 2.504 & 2.496 & $2.504[30,31]$ \\
$c_{\perp}(\AA)$ & & 6.490 & $6.66[30-32]$ \\
$E_{b}(\mathrm{eV} /$ c.u. $)$ & -13.38 & -12.826 & $-13.322[35]$ \\
$B(\mathrm{GPa})$ & & $30.1,26[33]$ & $36.7[30], 25.6[32]$ \\
$B_{2 D}(\mathrm{~N} / \mathrm{m})$ & 164 & $164[33], 280[34]$ & \\
$C_{11,2 D}(\mathrm{~N} / \mathrm{m})$ & 277 & $293[34]$ & \\
$\mu_{2 D}(\mathrm{~N} / \mathrm{m})$ & 113 & $114[34]$ & \\
$E_{2 D}(\mathrm{~N} / \mathrm{m})$ & 267 & $278[34]$ & \\
$\nu_{2 D}(\mathrm{~N} / \mathrm{m})$ & 0.186 & $0.225[34], 0.2[17]$ & \\
$\boldsymbol{c}$-BN & & & $3.615[36,37]$ \\
$a(\AA)$ & 3.5575 & 3.593 & $-13.49[35]$ \\
$E_{b}(\mathrm{eV} / \mathrm{c} . u)$. & -13.49 & -12.94 & $369-400[37,38]$ \\
$B(\mathrm{GPa})$ & 402 & 395 & $820[38]$ \\
$C_{11}(\mathrm{GPa})$ & 638 & 803 & $480[38]$ \\
$C_{44}(\mathrm{GPa})$ & & 475 & \\
$C_{44}^{0}(\mathrm{GPa})$ & 553 & 591 & \\
\hline \hline
\end{tabular}


FCC lattices are sufficiently higher in energy to make these structures safely unstable. In the DMol ab initio calculations, the $\mathrm{N}_{3}$ triangular cluster could not be geometrically optimized due to instability, but in Ref. [26], this cluster was found to be $1.37 \mathrm{eV} /$ atom less stable than linear $\mathrm{N}_{3}$, which is roughly reproduced by our potential.

Table III shows the comparison for the BN compound structures. Almost all reference properties, including the elastic moduli, are rather well reproduced by BN-ExTeP, with $c$-BN correctly described as the most stable BN polymorph at the given stoichiometry.

The parametrization was performed such that the (in-plane) lattice parameter for $h$-BN exactly matches the experimental value, a property which is desirable for studying heterostructures made of $h$-BN and graphene with the correct lattice mismatch between the two materials, required, e.g., for an accurate description of moiré pattern formation [27]. Note that in LAMMPS, BN-ExTeP can be used in conjunction with a Tersoff potential for carbon.

The lattice parameter for $c$-BN is about $1.6 \%$ too small, which in general is not a problem. We note, however, that for applications to $c$-BN requiring a more accurate lattice parameter, the lattice parameter can be adjusted by a factor $s_{a}$ without changing the binding energies by scaling the Tersoff parameters $\beta$ and $r_{0}$ to $\tilde{\beta}=\beta / s_{a}$ and $\tilde{r}_{0}=s_{a} r_{0}$, respectively, as can be shown analytically. Obviously, this scaling reduces the interatomic distances in all BN structures by the same factor, leading to a less accurate lattice parameter for $h$-BN, which, however, is irrelevant for applications to $c$-BN. It also changes the elastic moduli by a factor of the order of $1 / s_{a}^{2}$, but as long as $s_{a}$ is close to one, as it is the case here, these changes typically remain rather small.

The experimental bulk modulus of $3 \mathrm{D} h-\mathrm{BN}$ is about one order of magnitude smaller than that for $c$-BN due to the high compressibility in the $c_{\perp}$ direction related to the relatively weak interactions between the $h$-BN layers. The bulk modulus $B$ according to $\mathrm{BN}-\mathrm{ExTeP}$ vanishes as it does not describe interlayer interactions. More relevant for us, however, are the in-plane or $2 \mathrm{D}$ elastic moduli $B_{2 D}$ and $C_{11,2 D}$. For a strictly $2 \mathrm{D}$, isotropic material these are related to the $2 \mathrm{D}$ shear modulus $\mu_{2 D}$, the 2D Young's modulus $E_{2 D}$, and the in-plane Poisson's ratio $v_{2 D}$ by the relations:

$$
\begin{aligned}
\mu_{2 D} & =C_{11,2 D}-B_{2 D}, \quad E_{2 D}=\frac{4 B_{2 D} \mu_{2 D}}{B_{2 D}+\mu_{2 D}} \text { and } \\
\nu_{2 D} & =\frac{B_{2 D}-\mu_{2 D}}{B_{2 D}+\mu_{2 D}},
\end{aligned}
$$

respectively. Using these relations, we find a Poisson's ratio $v_{2 D} \simeq 0.19$, which compares well to the $a b$ initio value of 0.2 reported in Ref. [17]. In particular, the uniaxial elastic modulus of $C_{11}=277 \mathrm{~N} / \mathrm{m}$ for $h$-BN is in much better agreement with the $a b$ initio value than the too large value of $C_{11}=396 \mathrm{~N} / \mathrm{m}$ according to the earlier Tersoff potential $[7,16]$, that leads to a negative Poisson's ratio equal to $v=-0.17$ using the relations in Eq. (15).

Not shown in Table III is that BN-ExTeP also gives reasonable values for the lattice parameters and binding energies of the rock-salt and BCC structures. According to $a b$ initio calculations [16] these unstable rock-salt and BCC BN compounds are about 3.5 and $7.8 \mathrm{eV} / \mathrm{c} . \mathrm{u}$. (eV per $\mathrm{BN}$ chemical unit) less stable than $c$-BN, respectively, while BN-ExTeP yields values of 3.88 and $9.33 \mathrm{eV}$, respectively, for these energy differences, the somewhat larger disagreement for the BCC BN compound being due to the effect of the cutoff. The lattice parameter of $3.535 \AA$ for rock salt and $2.299 \AA$ for the BCC $\mathrm{BN}$ compound according to $\mathrm{BN}-\mathrm{ExTeP}$ correspond well to the DFT values of $3.474 \AA$ and $2.32 \AA$, respectively.

The pure and compound systems considered in this section do not involve any nonzero correction term $F_{I J}$. In fact, the correction terms $F_{B B}(0,0)$ and $F_{N N}(0,0)$ involved in the pure systems and the terms $F_{B N}(2,2)$ and $F_{B N}(3,3)$ involved in $h$-BN and $c$-BN, respectively, are all taken equal to zero. All other correction terms are adjusted to optimize the description of defects in $h$-BN and $c$-BN and of small BN clusters, as detailed in the next section.

\section{B. Bond order correction}

The correction term $F_{i j}$ is a natural, first order correction to the bond order for binary systems, involving only nearest neighbors and their chemical identities. This term considerably improves the description of the formation energy and geometry (bond distances) of a whole series of defects and a number of small BN molecules. Clearly, this improvement comes at the cost of additional parameters, but it hardly affects the efficiency of the calculations, as it does not extend the environment involved in computing the bond order, which remains restricted to the nearest neighbors.

The correction term parameters are listed in Table IV. In Table $\mathrm{V}$ we describe the defects in $h$-BN and $c$-BN that we considered. For $h$-BN, these defects and selected bond lengths are illustrated in Fig. 1. The Stone-Wales (SW) defect in $h$-BN is the BN analog of the SW defect for graphene [39]. There are two local minima for the SW defect in $h$-BN, with different geometries around the defect as illustrated in the bottom panel of Fig. 1. As for graphene [40], the most stable structure is SW-1, a sinelike buckling around the defect. The antisite (AS) defect is created by swapping the chemical identity of

TABLE IV. Values of $F_{I J}$. For integer arguments with $N_{i j}^{\bar{I}}$ or $N_{j i}^{\bar{J}} \geqslant 4, F_{I J}$ is zero.

\begin{tabular}{crcccc}
\hline \hline & $N_{i j}^{\bar{B}} \backslash N_{j i}^{\bar{B}}$ & 0 & 1 & 2 & \multicolumn{1}{c}{3} \\
\hline \multirow{4}{*}{$F_{B B}$} & 0 & 0.0000 & 0.0054 & 0.0182 & -0.0034 \\
& 1 & 0.0054 & 0.0100 & 0.0062 & 0.0154 \\
& 2 & 0.0182 & 0.0062 & 0.0154 & -0.0390 \\
& 3 & -0.0034 & 0.0154 & -0.0390 & -0.1300 \\
& $N_{i j}^{\bar{N}} \backslash N_{j i}^{\bar{N}}$ & 0 & 1 & 2 & 3 \\
$F_{N N}$ & 0 & 0.0000 & -0.0282 & -0.0018 & -0.0004 \\
& 1 & -0.0282 & 0.0200 & 0.0180 & 0.0146 \\
& 2 & -0.0018 & 0.0180 & 0.0306 & 0.0060 \\
& 3 & -0.0004 & 0.0146 & 0.0060 & 0.0000 \\
$F_{B N}$ & $N_{i j}^{\bar{B}} \backslash N_{j i}^{\bar{N}}$ & 0 & 1 & 2 & 3 \\
& 0 & 0.0170 & 0.0078 & 0.0000 & -0.0860 \\
& 1 & -0.0090 & 0.0090 & -0.0068 & -0.0338 \\
& 2 & 0.0000 & -0.0198 & 0.0000 & -0.0084 \\
& 3 & -0.0750 & -0.0168 & -0.0138 & 0.0000 \\
\hline \hline
\end{tabular}


TABLE V. Considered defects in $h$-BN and $c$-BN with involved correction terms $F_{I J}$. Formation energies $E_{f}$ in $\mathrm{eV}$ as found with the classical potentials of Albe and Möller [7], Kınacı et al. [10], and from this work are compared to DFT calculations with DMol from Ref. [16] and those calculated using SIESTA by us.

\begin{tabular}{|c|c|c|c|c|c|c|}
\hline \multirow[b]{2}{*}{$h-\mathrm{BN}$} & \multirow[b]{2}{*}{$F_{I J}$ involved } & \multicolumn{3}{|c|}{$E_{f}($ Classical Potentials $)$} & \multicolumn{2}{|c|}{$E_{f}(\mathrm{DFT})$} \\
\hline & & {$[7]$} & {$[10]$} & BN-ExTeP & [16] & SIESTA \\
\hline AS & $F_{B B}(1,2), F_{N N}(1,2), F_{B N}(1,2), F_{B N}(2,1)$ & 7.3 & 9.3 & 8.1 & 8.1 & 8.52 \\
\hline SW-1 & $F_{B B}(2,2), F_{N N}(2,2), F_{B N}(1,1), F_{B N}(1,2), F_{B N}(2,1)$ & 9.18 & 6.44 & 7.22 & & 7.20 \\
\hline SW-2 & $F_{B B}(2,2), F_{N N}(2,2), F_{B N}(1,1), F_{B N}(1,2), F_{B N}(2,1)$ & 9.12 & 6.74 & 7.43 & & 7.34 \\
\hline B sub & $F_{B B}(0,2), F_{B N}(1,2)$ & 5.0 & 6.8 & 5.7 & 5.7 & 6.50 \\
\hline N sub & $F_{N N}(0,2), F_{B N}(2,1)$ & 5.8 & 7.1 & 9.1 & 9.1 & 8.98 \\
\hline B vac & $F_{B N}(2,1)$ & 4.7 & 4.7 & 10.4 & 10.4 & 10.55 \\
\hline $\mathrm{N}$ vac & $F_{B N}(1,2)$ & 5.6 & 5.6 & 7.3 & 7.3 & 6.25 \\
\hline Interc. B1 & $F_{B B}(1,3), F_{B N}(2,3)$ & 3.4 & 3.6 & 7.3 & 7.3 & \\
\hline Interc. B2 & $F_{B B}(2,3), F_{B N}(1,3), F_{B B}(2,3)$ & 4.3 & 5.6 & 8.0 & - & \\
\hline Interc. N1 & $F_{N N}(1,3), F_{B N}(3,2)$ & 3.5 & 3.3 & 6.6 & - & \\
\hline Interc. N2 & $F_{N N}(2,3), F_{B N}(3,1), F_{B N}(3,2)$ & 3.2 & 0.5 & 7.3 & 7.3 & \\
\hline \multicolumn{7}{|l|}{$c$-BN } \\
\hline AS & $F_{B B}(1,3), F_{N N}(1,3), F_{B N}(2,3), F_{B N}(3,2)$ & 7.0 & 10.2 & 9.1 & 9.1 & \\
\hline B sub & $F_{B B}(0,3), F_{B N}(2,3)$ & 2.1 & 5.5 & 9.1 & 9.1 & \\
\hline $\mathrm{N}$ sub & $F_{N N}(0,3), F_{B N}(3,2)$ & 5.8 & 9.3 & 10.2 & 10.2 & \\
\hline B vac & $F_{B N}(3,2)$ & 3.4 & 2.6 & 9.2 & 9.2 & \\
\hline $\mathrm{N}$ vac & $F_{B N}(2,3)$ & 2.5 & 3.5 & 7.3 & 7.3 & \\
\hline
\end{tabular}

two nearest neighbor atoms. The $\mathrm{B}(\mathrm{N})$ substitutional defects are created replacing an individual $N(B)$ atom by $B(N)$. The intercalation defects are created by putting an additional atom between two layers of $h-\mathrm{BN}$ in AA stacking, where $\mathrm{N}$ atoms of the first layer are above $\mathrm{B}$ in the second. The intercalated atom is then chemically bound to both layers, being either vertically intercalated between B and N (B1 or $\mathrm{N} 1$ ), or diagonally between two atoms with chemical identities different from itself (B2 or N2).
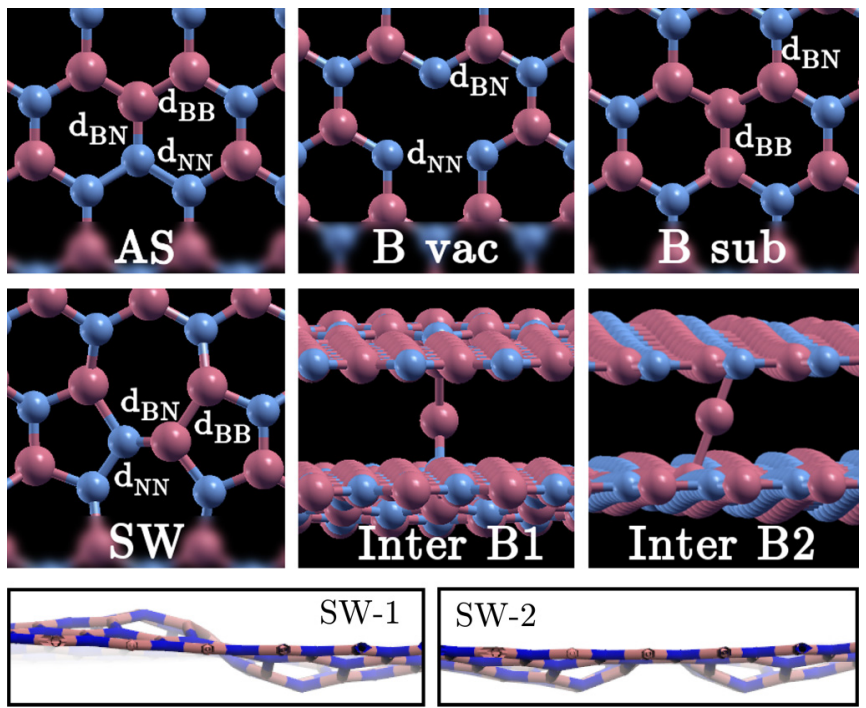

FIG. 1. Point defects in $h$-BN and distances considered in Table VI. For the Stone-Wales defect side views of the antisymmetric (SW-1) and symmetric (SW-2) realizations are shown in the bottom panel.
The defect formation energies $E_{f}$ of our neutral defects are calculated as $[16,41,42]$ :

$$
E_{f}=E_{D}-n_{B} \mu_{B}^{B N}-n_{N} \mu_{N}^{B N},
$$

where $E_{D}$ is the total energy of the defected system, $n_{B}$ and $n_{N}$ are the number of $\mathrm{B}$ and $\mathrm{N}$ atoms in the supercell, respectively, and $\mu_{B}^{B N}$ and $\mu_{N}^{B N}$ their chemical potentials in the BN compound phase. Following Ref. [16], Eq. (16) can be rewritten to:

$E_{f}=E_{D}-\frac{1}{2}\left(n_{B}+n_{N}\right) \mu_{B N}-\frac{1}{2}\left(n_{B}-n_{N}\right)\left(\mu_{B}-\mu_{N}\right)$,

where $\mu_{B N}=\mu_{B}^{B N}+\mu_{N}^{B N}=\mu_{B}+\mu_{N}+\Delta H_{f}$ is the chemical potential per BN pair in the compound bulk phase, $\mu_{B}$ and $\mu_{N}$ are the chemical potentials of $\mathrm{B}$ and $\mathrm{N}$ in pure component reference phases, and $\Delta H_{f}$ is the formation enthalpy. In the present case, $\mu_{B N}$ is just the ground state binding energy, equal to -13.38 and $-13.49 \mathrm{eV} / \mathrm{c} . \mathrm{u}$. for $h-\mathrm{BN}$ and $c$-BN, respectively, according to BN-ExTeP (see Table III). Obviously, for the calculation of the $a b$ initio formation energies, the respective $a b$ initio ground state binding energies were used. It can be shown that, in thermodynamic equilibrium, $\mu_{B}-\mu_{N}$ is approximately equal to the difference in the binding energy per atom of the pure component reference phases. We have chosen the reference phases to be solid $\alpha$ phase for boron and diatomic $\mathrm{N}_{2}$ for nitrogen, with binding energies 5.81 and $4.92 \mathrm{eV} /$ atom, respectively, yielding $\mu_{B}-\mu_{N}=-0.89$ $\mathrm{eV} /$ atom in thermodynamic equilibrium.

Each defect involves one or more correction term values for integer arguments, specified in the second column of Table V, which are adjusted to optimize the defect description. The resulting defect formation energies for optimized geometries in Table $\mathrm{V}$ are globally in very good agreement with those from DFT calculations [16] and significantly improve the formation energies with respect to previous potentials from 
TABLE VI. Interatomic distances for defects in $h$-BN, compared to $a b$ initio values calculated using the SIESTA code.

\begin{tabular}{lccc}
\hline \hline Structure & $d_{B B}(\AA)$ & $d_{N N}(\AA)$ & $d_{B N}(\AA)$ \\
\hline AS & $1.60(1.61)$ & $1.44(1.44)$ & $1.36(1.36)$ \\
SW-1 & $1.60(1.68)$ & $1.41(1.46)$ & $1.37(1.36)$ \\
SW-2 & $1.60(1.68)$ & $1.40(1.46)$ & $1.37(1.35)$ \\
B sub & $1.55(1.63)$ & & $1.47(1.45)$ \\
N sub & & $1.53(1.49)$ & $1.51(1.44)$ \\
B vac & & $2.60(2.58)$ & $1.48(1.40)$ \\
N vac & $2.66(2.27)$ & & $1.43(1.47)$ \\
\hline \hline
\end{tabular}

Refs. $[7,10]$ listed in the same table. They are all in the right order, with most of the defects in $h$-BN having formation energies between 5 and $10 \mathrm{eV}$ and the B substitutional defect having the lowest formation energy. There is a small energy difference between the two SW defects, assigning the correct most stable configuration when compared to the $a b$ initio calculations. BB-ExTeP predicts higher stability of the B1 and $\mathrm{N} 1$ intercalation defects compared to the B2 and N2 types, respectively. For the $\mathrm{B}$ interaction this seems in agreement with $a b$ initio calculations but not for the $\mathrm{N}$ intercalation. We note that the formation energy of the B2 and N1 intercalation defects could not be determined in the DMol calculations as they transformed into the more stable B1 and N2 structures, respectively, during geometrical optimization.

Experimentally observed vacancy defects, created by an electron beam, have been associated with only one of the two sublattices [13], which was later identified as a predominance of B monovacancies [43]. This is in contrast with its higher formation energy (Table V). It should be noticed, however, that although the formation energy provides a valuable tool in the construction of a potential and the comparison of theoretical methods, it is a difficult quantity to relate directly to experiments as its value depends on the chosen reference states, that may not reflect the experimental environment, and does not consider kinetic effects nor the dynamical stability, determined, e.g., by energies barriers. In the present case, the abundance of $\mathrm{B}$ monovacancies may be due to the lighter mass of $\mathrm{B}$, making it more easy to excite $\mathrm{B}$ atoms to a state where they have a velocity large enough for escape from the lattice.

Bond distances around a number of important defects in $h$-BN are given in Table VI and compared to the values in parenthesis from $a b$ initio calculations using the SIESTA code. Differences with $a b$ initio values are relatively small and not larger than $5 \%$ in all cases except for the $\mathrm{N}$ vacancy, which is overall satisfactory.

Apart from the defects, also linear and triangular $B_{2} N$ and $B N_{2}$ clusters were used as references for the adjustment of a number of correction terms $F_{i j}$. These are listed in Table VII, together with their binding energy per atom $E_{b}$ and bond distances after geometrical optimization, compared to the DMol results. The table shows that the binding energies for these small binary clusters are substantially smaller that those in the stable compound bulk phases, implying that such clusters are relatively unstable in a $\mathrm{BN}$ environment, which is correctly reproduced by the classical potential. Therefore, trying to reduce the somewhat larger disagreements with the DMol values for the triangular clusters was not given a high priority.
TABLE VII. Linear and triangular three atom binary B-N clusters considered for adjustment of the specified correction terms $F_{I J}$, their binding per atom and associated bond distances, compared to the DMol values [16] in parenthesis.

\begin{tabular}{lccc}
\hline \hline Structure & $F_{i j}$ & $E_{b}(\mathrm{eV} /$ atom $)$ & $d_{I J}(\AA)$ \\
\hline lin. BBN & $F_{B B}(1,0)$ & $3.31(3.32)$ & $d_{B B}=1.59(1.55)$ \\
& $F_{B N}(0,0)$ & & $d_{B N}=1.30(1.31)$ \\
lin. BNB & $F_{B N}(0,1)$ & $4.09(4.10)$ & $d_{B N}=1.34(1.32)$ \\
tr. $\mathrm{B}_{2} \mathrm{~N}$ & $F_{B B}(1,1)$ & $3.42(3.93)$ & $d_{B B}=1.60(1.68)$ \\
& $F_{B N}(0,1)$ & & $d_{B N}=1.62(1.35)$ \\
lin. BNN & $F_{N N}(1,0)$ & $3.52(3.52)$ & $d_{N N}=1.45(1.19)$ \\
& $F_{B N}(0,0)$ & & $d_{B N}=1.30(1.33)$ \\
lin. NBN & $F_{B N}(1,0)$ & $3.37(3.37)$ & $d_{B N}=1.40(1.36)$ \\
tr. $\mathrm{BN}_{2}$ & $F_{N N}(1,1)$ & $2.44(3.56)$ & $d_{N N}=1.74(1.34)$ \\
& $F_{B N}(1,0)$ & & $d_{B N}=1.46(1.43)$ \\
\hline \hline
\end{tabular}

We note that the $h$-BN nanoribbon edge formation energies are also reasonably well reproduced without being considered during the parametrization. We find formation energies of 9.0 and $12.2 \mathrm{eV} / \mathrm{nm}$ for the armchair and zigzag edges of $h-\mathrm{BN}$, respectively, compared to DFT-PBE values of 7.6 and $11.2 \mathrm{eV} / \mathrm{nm}$ [44].

\section{APPLICATION TO PRISTINE $h$-BN}

To introduce the study of the elastic moduli of defective $h$-BN presented in the next section, we first comment on some basic properties of pristine $h$-BN as obtained by molecular dynamics (MD) simulations with BN-ExTeP. Some of these properties were also investigated in a previous study $[45,46]$ based on an earlier BN Tersoff potential [7].

For this and the following section we performed a series of classical molecular dynamics simulations at constant temperature and constant-pressure (NPT). The equations of motion including thermostat and barostat [47] are integrated with a timestep of $1 \mathrm{fs}$. For both the Nosé-Hoover thermostat and barostat, we used a coupling constant (damping parameter) of 1 ps. The barostat is only applied to the in-plane dimensions of the membrane. We consider a sheet of $h$-BN consisting of 37888 atoms in a periodic cell of $L_{x} \times L_{y}=32.4 \times 32.6 \mathrm{~nm}^{2}$ $(74 \times 128$ orthorhombic unit cells). The $x$ and $y$ directions here correspond to zigzag and armchair axes, respectively. The perpendicular cell size $\left(L_{z}=4 \mathrm{~nm}\right)$ is large enough to avoid interaction between periodic images of the sheet.

We first examine the temperature dependence of the 2D lattice parameter. For this, we performed 48 independent MD simulations at temperatures up to $4000 \mathrm{~K}$. Each of these simulations ran for 200 ps of which the last 100 ps were used to obtain average lattice constants. The result is shown in the top panel of Fig. 2. The decrease of the lattice parameter is a consequence of thermal rippling. Unlike the situation for graphene, however, for which a minimum in $a$ corresponding to a compression of $\sim 0.2 \%$ was found at $900 \mathrm{~K}$ [48] from simulations based on the carbon potential LCBOPII [49], here $a$ does not have a minimum but decreases monotonously up to a temperature close to or beyond the melting temperature, where a compression of about $1 \%$ is reached. This behavior also deviates from the results for $h-\mathrm{BN}$ in Ref. [45] by 

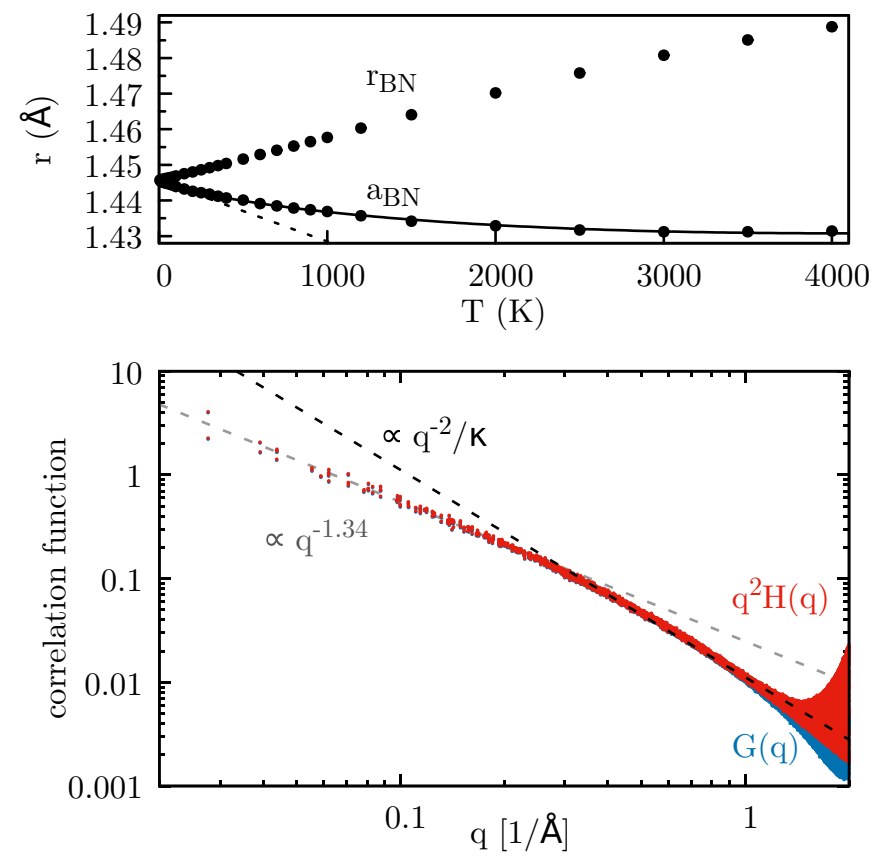

FIG. 2. (top) Average in-plane lattice parameter $a_{B N}=$ $\left(a / a_{0}\right) r_{N B, 0}$ and average B-N nearest neighbor distance $r_{N B}$ as a function of temperature, with $r_{N B, 0}$ the nearest neighbor distance at $T=0 \mathrm{~K}$ and $a_{0}=2.504 \AA$ the ground state lattice parameter. The dashed line is a linear fit up to $100 \mathrm{~K}, a=a_{0}(1+\alpha T)$ with $\alpha=-1.181 \times 10^{-5} \mathrm{~K}^{-1}$. A good fit over the whole range (solid line) is found with the form $a=a_{0}\left(1+\alpha_{1} T+\left(\alpha_{2} T\right)^{\xi}\right)$, with $\alpha_{1}=5.16 \times 10^{-4} \mathrm{~K}^{-1}, \alpha_{2}=5.204 \times 10^{-4}$, and $\xi=0.995$. (bottom) Normal-normal $[\mathrm{G}(\mathrm{q})]$ and height-height $\left[q^{2} H(q)\right]$ correlation functions at room temperature $T=300 \mathrm{~K}$. The harmonic regime $\propto q^{-2}$ (dashed line) yields a bending rigidity $\kappa=0.85 \mathrm{eV}$. For $q \rightarrow 0$ we find a power law with exponent $-(2-\eta)$ with $\eta \sim 0.66$.

simulations based on the earlier BN potential by Albe [7] and in Ref. [50] based on the potential of Kinaci [10]. In these previous studies, minima in $a$ were found at $1500 \mathrm{~K}$ and $2000 \mathrm{~K}$, respectively, corresponding to a compression of about $0.5 \%$ in both cases. A negative thermal expansion coefficient is a common property of all membranes in the quasiharmonic approximation [51]; it follows from negative (and divergent at small wave vectors) microscopic Grüneisen parameters for the acoustic flexural mode [52] and has also been measured experimentally for graphene [53]. The change of its sign at high enough temperatures, which is also predicted for graphene [48], is an anharmonic effect beyond the quasiharmonic approximation and beyond the macroscopic phenomenological theory of membranes, namely it is determined by phononphonon interactions over the whole Brillouin zone [54]. The temperature at which this change in sign appears, however, can be higher than the melting temperature, as seems to be the case for $h$-BN according to BN-ExTeP.

In contrast to the 2D lattice parameter, which decreases, the B-N nearest neighbor distance monotonously increases with temperature, as also shown in Fig. 2. This expected, approximately linear increase was also noticed for graphene in Ref. [55].

We also considered the structure of the thermal rippling at $T=300 \mathrm{~K}$. For this we did a separate MD simulation of
$1.25 \mathrm{~ns}$, of which an initial part was used for thermalization and the remaining part for averaging. From these simulations we computed the Fourier transform of the normal-normal correlation function, $G(q)=\left\langle\left|\mathbf{n}_{q}\right|^{2}\right\rangle=N^{-1}\left\langle\left|\sum_{i}^{N} \hat{\mathbf{n}}_{i} \exp \left(i \mathbf{q} \mathbf{r}_{i, 0}\right)\right|^{2}\right\rangle$ and height-height correlation function $H(q)=\left\langle\left|h_{q}\right|^{2}\right\rangle=$ $N^{-1}\left\langle\left|\sum_{i}^{N} h_{i} \exp \left(i \mathbf{q r}_{i, 0}\right)\right|^{2}\right\rangle$ with $\hat{\mathbf{n}}_{i}$ the unit vector normal to the surface at site $i, h_{i}$ the height at site $i, \mathbf{q}$ the wave vector in Fourier space, and $\left\{\mathbf{r}_{i, 0}\right\}$ the ground state atomic positions. These were calculated as done for graphene in Ref. [56] and are shown in the bottom panel of Fig. 2. It holds that $G(q)=q^{2} H(q)$. In the harmonic approximation, i.e., for large $q$ (roughly from 0.3 to $1 \AA^{-1}$ in the present case), we have $H(q)=\rho k_{B} T /\left(\kappa q^{4}\right)$ and $G(q)=\rho k_{B} T /\left(\kappa q^{2}\right)$, with $\rho$ the $2 \mathrm{D}$ density in atoms $/ \AA^{2}$ and $\kappa$ the bending rigidity. With this we find $\kappa=0.85 \mathrm{eV}$ compared to $0.96 \mathrm{eV}$ from ab initio calculations [57] and 0.86-1 eV as calculated using the potential by Kinac1 et al. [10] in Ref. [58]. According to these results, freestanding $h$-BN is about as rigid as freestanding graphene, for which a bending rigidity of $\kappa=1.1 \mathrm{eV}$ was found at $T=300 \mathrm{~K}$ [59] using the LCBOPII interatomic potential for carbon [49], the latter value being slightly smaller than $\kappa=1.2 \mathrm{eV}$ obtained experimentally via the phonon spectrum of graphite [60].

In the long wavelength limit, due to strong anharmonic coupling between in-plane modes and out-of-plane flexural modes, height fluctuations are suppressed and the correlation function behaves as $G(q)=\propto q^{2-\eta}$, with $\eta$ a nontrivial critical exponent. From our calculated $G(q)$ we find $\eta \sim 0.66$, compared to 0.85 found for graphene [56]. While this difference may appear to be significant, it should be noted that the value of $\eta$ obtained from simulations can depend on simulation settings and tends to converge very slowly. We estimate the error in our extracted exponent to be approximately of the order of this difference, so that the supposed universality of this behavior is neither contradicted nor confirmed.

We investigated the elastic behavior of pristine $h$-BN under uniaxial strain by running simulations at a temperature $T$ near $0 \mathrm{~K}$. The results are summarized in Fig. 3 and allow us to recalculate the elastic modulus and Poisson's ratio independently and verify the consistency of Eq. (15) in this way. Note that the $E_{2 D}$ and $v$ in Table III were calculated from $B_{2 D}$ and $C_{11}$ using Eq. (15). The top-left figure shows the applied, fixed $L_{x}$ and free $L_{y}$ cell size dimensions. The sample used in this simulation is initially compressed to $99.5 \%$ of its equilibrium value along the $x$ direction. After $2 \mathrm{~ns}$ equilibration, the sample is then gradually extended to $100.5 \%$ during $10 \mathrm{~ns} \mathrm{MD}$ in steps of $0.1 \%$. The cell size in the $y$ direction is kept free and is found to decrease, indicating a positive Poisson's ratio equal to 0.19 in agreement with the value in Table III.

In the bottom-left panel of Fig. 3, the stress component in the $x$ direction, $\sigma_{x x}$, as a function of simulation time is shown. On the right-hand side, time averages of the energy and stress as a function of the fixed strain are shown. For compressive strain, $\sigma_{x x}$ remains very small as a result of induced static corrugation. The same can be seen from the energy increase at negative strain (top-right panel), which is evidently not quadratic, as for positive (tensile) strain, but displays a much smaller, near-constant increase in average 

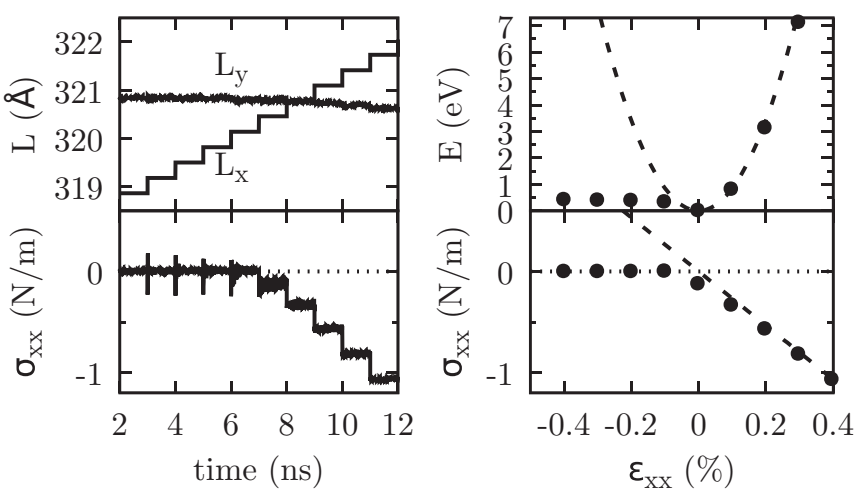

FIG. 3. Summary of the results from simulations of pristine $h$-BN under uniaxial applied strain $\epsilon_{x x}$ in the $x$ direction, varied in steps from 0.95 to 1.05 as described in the text, at a temperature near $0 \mathrm{~K}$ with size fluctuations in the $y$ direction. The top left panel shows how $L_{x}$ is varied in steps to induce the strain and the monitored $L_{y}$ during the simulation. The bottom left panel shows the monitored $x x$ component of the stress tensor $\sigma_{x x}$. The right panels show the average energy per atom (top) and the average $\sigma_{x x}$ as a function of the applied strain $\epsilon_{x x}$. The dashed line in the right top panel corresponds to a $E_{2 D}=267 \mathrm{~N} / \mathrm{m}$.

potential energy illustrative of strong anharmonicity. The simulation data for positive strain part $\left(\epsilon_{x x}>0\right)$ are very well fitted by the quadratic curve (dashed line) yielding a the 2D Young's modulus of $E_{2 D}=267 \mathrm{~N} / \mathrm{m}$, consistent with Table III.

\section{APPLICATION TO DEFECTIVE $h$-BN}

To study the effect of defects on the elastic properties of $h$-BN, we consider six defect types. These are the Stone-Wales (SW) and antisite (AS) defects, vacancies (B and N) and substitutional defects ( $\mathrm{B}$ and $\mathrm{N}$ ). Here we notice that the results presented in this section have been obtained from a slightly different parametrization of our potential [61]. This does not change qualitatively the results and has a negligible quantitative effect as we verified.

We use the same sample size as considered for pristine $h$-BN in the preceding section. For each defect type we then consider seven defect densities up to $0.7 \mathrm{~nm}^{-2}$ corresponding to $38,76,114,152,228,304$, and 760 defects and for each of these densities we consider between five and eight different randomly generated defect distributions. For each of these samples we then first perform a thermal optimization. To subsequently determine the elastic moduli, we used the same procedure as described earlier for pristine $h$-BN.

Our thermal optimization procedure is done in the NPT ensemble at $P=0$ and consists of a 200 ps heating from 100 to $1000 \mathrm{~K}$, thermalization at $1000 \mathrm{~K}$ for $2 \mathrm{~ns}$, cooling down to $10 \mathrm{~K}$ in $300 \mathrm{ps}$, followed by a conjugate gradient minimization. The final structures gives us the equilibrium lattice parameters and the defect formation energies including defect-defect interactions.

The results are shown in Fig. 4. The panels in the first row show the relative cell sizes in the $x$ direction for the optimized structures. All defects considered here result in a

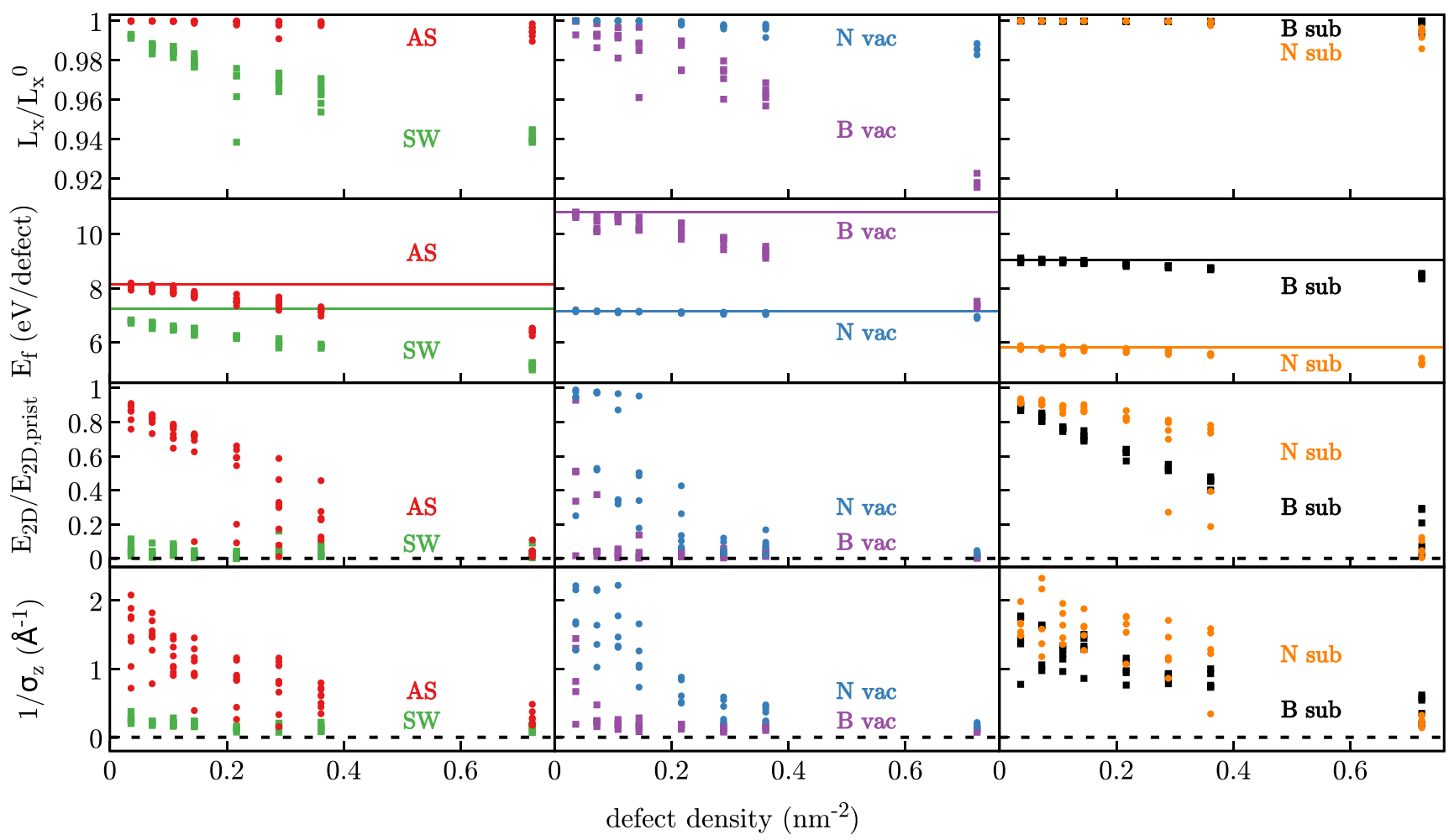

FIG. 4. From top to bottom, rows of panels show the static strain $L_{x} / L_{x}^{0}$, average defect formation energies $E_{f}$, relative elastic moduli $E_{2 D} / E_{2 D \text {,prist }}$, and inverse corrugation $1 / \sigma_{z}$, with $\sigma_{z}$ the average height fluctuation of the annealed samples, as a function of the defect concentration. Multiple symbols are results for different random realizations. Defect types are as indicated in the graph. In the plots of the $E_{f}$, horizontal lines indicate the isolated defect formation energy for reference. 

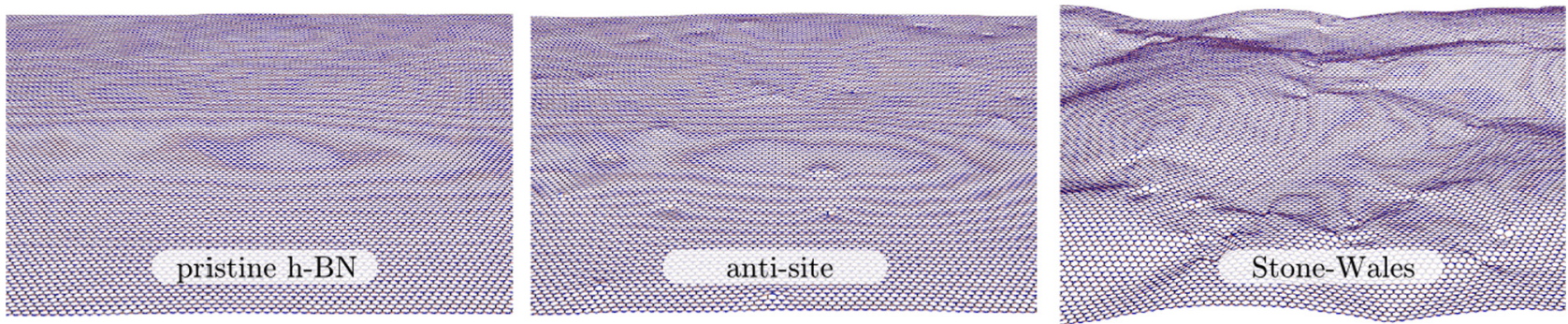

FIG. 5. Illustration of selected structures at $T=0 \mathrm{~K}$. The AS and SW defect densities (middle and right panel) are $0.036 \mathrm{~nm}^{-2}$, the lowest defect densities considered here.

decrease of the cell size with respect to pristine $h$-BN. SW defects and B vacancies show the most obvious decrease, being roughly linear as a function of the defect density. This can be understood as a result of static corrugation. Contrary to thermal rippling, static corrugation does not disappear at low temperatures because it is the result of local curvature induced by the defects. These out-of-plane distortions lead to a reduction of the in-plane lattice constant. As an example, the optimized structure of pristine $h$-BN is compared to those of $h$-BN with AS and SW defects in Fig. 5 . The pristine lattice is entirely flat in the low temperature limit. Antisite defects induce small localized distortions while Stone-Wales defects lead to much stronger and longer-ranged distortions. As a result, the samples with SW defects are indeed strongly corrugated and their associated cell sizes are significantly reduced. Substitutional defects present very little corrugation and correspondingly have nearly unchanged lattice constants.

The second row of Fig. 4 shows the corresponding defect formation energies (in $\mathrm{eV}$ per defect). Note that, while all are drawn on the same scale, the absolute values of formation energies depend on the conventions used for Eqs. (16) and (17), related to the choice of the pure component reference systems. The SW defects and B vacancies, as well as the AS defects, show a strong linear decrease in formation energy as a function of defect density. This indicates that defects that induce strong corrugation highly prefer being in close proximity over being far apart, a behavior also observed for graphene [62,63]. On the contrary, $\mathrm{N}$ vacancies and substitutional defects appear to interact only weakly and thus do not significantly affect formation energies. Only at elevated defect densities a small energy gain can be noticed for the vacancy configurations, which is mostly the result of reconstructions during the annealing procedure.

The third row of Fig. 4 shows the calculated Young's moduli relative to the value for pristine $h$-BN. In all cases $E_{2 D}$ is significantly affected by the defects, albeit in qualitatively different ways. SW defects and B vacancies dramatically decrease the Young's modulus, even at low concentrations. Also this can be understood from the corrugation of the sample. When the corrugated sample is stretched, the energy required to flatten the system is much smaller than if chemical bonds were to be stretched directly as in the case of (flat) pristine $h$-BN. The AS and substitutional vacancies lead to a much more gradual and approximately linear decrease in elastic moduli. Finally, $\mathrm{N}$ vacancies show again a different behavior. At low densities their elastic moduli are nearly unaffected, but as the concentration increases, defect reconstructions start to occur and the elastic moduli suddenly drop similar to what was observed for SW defects and B vacancies despite being accompanied by a rather small change in average cell size. For vacancies in graphene, the Young's modulus has been observed to increase at concentrations below $\sim 0.1 \mathrm{~nm}^{-1}(0.2 \%)$ [64] Such an increase in $E_{2 D}$ can be the result of pre-straining created during the formation of vacancies, which suppresses anharmonic effects and by this the impact of anharmonicity on the effective elastic modulus [65] assuming a clamped boundary of the system. Our samples are not pre-strained because our simulations are at zero pressure and anharmonic effects due to thermal height fluctuations do not play a role at $T=0 \mathrm{~K}$.

To illustrate the strong correlation between the behavior of the elastic moduli and the static corrugation more explicitly, we have calculated the average height fluctuation, $\sigma_{z}^{2}=\langle(z-$ $\left.\langle z\rangle)^{2}\right\rangle$, which is shown in the last row of Fig. 4. Indeed, we find that the values for $\sigma_{z}$ vary similarly with defect concentration as the elastic moduli, consistent with the foregoing discussion.

\section{CONCLUSIONS AND PERSPECTIVES}

We have developed BN-ExTeP, an extended Tersoff potential for $\mathrm{B}, \mathrm{N}$, and $\mathrm{BN}$ describing a wide range of structures and being particularly accurate for the description of defects in $h$-BN and $c$-BN. The potential can be used efficiently to study the statistical mechanics of large (defected) $h$-BN sheets, knowing that large system size is very important for $2 \mathrm{D}$ systems. BN-ExTeP can also be a valuable tool for large-scale simulations involving chemical reactions and phase transformations, such as the melting and growth of $h$ - $\mathrm{BN}$ or $c$ $\mathrm{BN}$, as it correctly describes the bonding trends in $\mathrm{BN}$ systems.

Having demonstrated that the ExTeP scheme proposed here for $\mathrm{BN}$ systems can provide a significant improvement in the accuracy of bonding as a function of chemical environment, we believe that the here proposed correction term is a good candidate, not only to correct other Tersoff potential parametrizations for $\mathrm{BN}$ but for other binary systems as well. Moreover, this approach has the important quality of hardly affecting the efficiency of the computations with respect to the original Tersoff potential scheme.

We have implemented BN-ExTeP in LAMMPS and used this classical potential to study the effect of defects on the elastic properties of $h$-BN. We find that the calculated weakening of the elastic moduli parallels the defect-induced growth of static corrugation. 


\section{ACKNOWLEDGMENTS}

The research leading to these results received funding from the Foundation for Fundamental Research on Matter (FOM), part of the Netherlands Organisation for Scientific Research (NWO). The work was carried out on the Dutch national e-infrastructure with the support of SURF Cooperative. This project has received funding from the European Union's Horizon 2020 research and innovation programme under Grant Agreement No. 696656 - GrapheneCore1. We thank P. G. Steeneken and G. Slotman for useful comments and A. Loiseau and C. Bichara for supporting this project.
[1] A. K. Geim and I. V. Grigorieva, Nature (London) 499, 419 (2013).

[2] C. R. Dean, A. F. Young, I. Meric, C. Lee, L. Wang, S. Sorgenfrei, K. Watanabe, T. Taniguchi, P. Kim, K. L. Shepard, and J. Hone, Nat. Nanotechnol. 5, 722 (2010).

[3] L. Britnell, R. V. Gorbachev, R. Jalil, B. D. Belle, F. Schedin, A. Mishchenko, T. Georgiou, M. I. Katsnelson, L. Eaves, S. V. Morozov, N. M. R. Peres, J. Leist, A. K. Geim, K. S. Novoselov, and L. A. Ponomarenko, Science 335, 947 (2012).

[4] K. Watanabe, T. Taniguchi, and H. Kanda, Nat. Mater 3, 404 (2004).

[5] L. Schué, B. Berini, A. C. Betz, B. Plaçais, F. Ducastelle, J. Barjon, and A. Loiseau, Nanoscale 8, 6986 (2016).

[6] R. Bourrellier, S. Meuret, A. Tararan, O. Stéphan, M. Kociak, L. H. G. Tizei, and A. Zobelli, Nano Lett. 16, 4317 (2016).

[7] K. Albe and W. Möller, Comput. Mater. Sci. 10, 111 (1998).

[8] K. Matsunaga, C. Fisher, and H. Matsubara, Jpn. J. Appl. Phys. 39, L48 (2000).

[9] C. Sevik, A. Kinaci, J. B. Haskins, and T. Çağın, Phys. Rev. B 84, 085409 (2011).

[10] A. Kınacı, J. B. Haskins, C. Sevik, and T. Çağın, Phys. Rev. B 86, 115410 (2012).

[11] M. R. Weismiller, A. C. T. van Duin, J. Lee, and R. A. Yetter, J. Phys. Chem. A 114, 5485 (2010)

[12] S. J. Pai, B. C. Yeo, and S. S. Han, Phys. Chem. Chem. Phys. 18, 1818 (2016).

[13] J. C. Meyer, A. Chuvilin, G. Algara-Siller, J. Biskupek, and U. Kaiser, Nano Lett. 9, 2683 (2009).

[14] C. Jin, F. Lin, K. Suenaga, and S. Iijima, Phys. Rev. Lett. 102, 195505 (2009).

[15] T. Pham, A. L. Gibb, Z. Li, S. M. Gilbert, C. Song, S. G. Louie, and A. Zettl, Nano Lett. 16, 7142 (2016).

[16] K. Albe, Computersimulationen zu Struktur und Wachstum von Bornitrid, Ph.D. thesis, Technische Universität Dresden, http://nbn-resolving.de/urn:nbn:de:bsz:d120-qucosa-30654 (1998).

[17] K. N. Kudin, G. E. Scuseria, and B. I. Yakobson, Phys. Rev. B 64, 235406 (2001).

[18] D. Sánchez-Portal and E. Hernández, Phys. Rev B 66, 235415 (2002).

[19] S. J. Plimpton, J. Comput. Phys. 117, 1 (1995).

[20] J. Tersoff, Phys. Rev. Lett. 56, 632 (1986); 61, 2879 (1988); Phys. Rev. B 38, 9902 (1988); 37, 6991 (1988).

[21] J. H. Los and A. Fasolino, Phys. Rev. B 68, 024107 (2003).

[22] J. M. Soler, E. Artacho, J. D. Gale, A. García, J. Junquera, P. Ordejón, and D. Sánchez-Portal, J. Phys.: Condens. Matter 14, 2745 (2002).

[23] J. P. Perdew, Phys. Rev. B 33, 8822 (1986); 34, 7406 (1986).

[24] N. Troullier and J. L. Martins, Phys. Rev. B 43, 1993 (1991).

[25] N. N. Greenwood, Boron (Pergamon Press, Lincoln, UK, 1975).
[26] J. Widany, T. Frauenheim, T. Köhler, M. Sternberg, D. Porezag, G. Jungnickel, and G. Seifert, Phys. Rev. B 53, 4443 (1996).

[27] M. M. van Wijk, A. Schuring, M. I. Katsnelson, and A. Fasolino, Phys. Rev. Lett. 113, 135504 (2014).

[28] R. Wycko, Crystal Structures (Interscience Publishers, New York, 1974).

[29] C. Kittel, Introduction to Solid State Physics, 8th ed. (Wiley, Hoboken, NJ, 2005).

[30] V. L. Solozhenko, G. Will, and F. Elf, Solid State Commun. 96, 1 (1995).

[31] R. W. Lynch and H. G. Drickamer, J. Chem. Phys. 44, 181 (1966).

[32] A. Bosak, J. Serrano, M. Krisch, K. Watanabe, T. Taniguchi, and H. Kanda, Phys. Rev. B 73, 041402 (2006).

[33] N. Ohba, K. Miwa, N. Nagasako, and A. Fukumoto, Phys. Rev. B 63, 115207 (2001).

[34] Q. Peng, Wei Ji, and Suvranu De, Computat. Mater. Sci. 56, 11 (2012).

[35] H. Neumann, Cryst. Res. Technol. 30, 910 (1995).

[36] E. Knittle, R. M. Wentzcovitch, R. Jeanloz, and M. L. Cohen, Nature (London) 337, 349 (1989).

[37] T. Sōma, A. Sawaoka, and S. Saito, Mater. Res. Bull. 9, 755 (1974).

[38] M. Grimsditch, E. S. Zouboulis, and A. Polian, J. Appl. Phys. 76, 832 (1994).

[39] A. J. Stone and D. J. Wales, Chem. Phys. Lett. 128, 501 (1986).

[40] J. Ma, D. Alfè, A. Michaelides, and E. Wang, Phys. Rev. B 80, 033407 (2009).

[41] S. Okada, Phys. Rev. B 80, 161404 (2009).

[42] W. Orellana and H. Chacham, Phys. Rev. B 63, 125205 (2001).

[43] K. Suenaga, H. Kobayashi, and M. Koshino, Phys. Rev. Lett. 108, 075501 (2012).

[44] R. Mukherjee and S. Bhowmick, J. Chem. Theory Comput. 7 , 720 (2011).

[45] G. J. Slotman and A. Fasolino, J. Phys.: Condens. Matter 25, 045009 (2013).

[46] During the present study we have realized that the results reported in Ref. [45] for defective structures are flawed by an error in the Tersoff parameters whereas they are correct for pristine $h$-BN.

[47] W. Shinoda, M. Shiga, and M. Mikami, Phys. Rev. B 69, 134103 (2004).

[48] K. V. Zakharchenko, M. I. Katsnelson, and A. Fasolino, Phys. Rev. Lett. 102, 046808 (2009).

[49] J. H. Los, L. M. Ghiringhelli, E. J. Meijer, and A. Fasolino, Phys. Rev. B 72, 214102 (2005).

[50] S. Thomas, K. M. Ajith, S. Chandra, and M. C. Valsakumar, J. Phys.: Condens. Matter 27, 315302 (2015).

[51] N. Mounet and N. Marzari, Phys. Rev. B 71, 205214 (2005). 
[52] P. L. de Andres, F. Guinea, and M. I. Katsnelson, Phys. Rev. B 86, 144103 (2012).

[53] D. Yoon, Y.-W. Son, and H. Cheong, Nano Lett. 11, 3227 (2011).

[54] M. I. Katsnelson and A. Fasolino, Acc. Chem. Res. 46, 97 (2013).

[55] M. Pozzo, D. Alfè, P. Lacovig, P. Hofmann, S. Lizzit, and A. Baraldi, Phys. Rev. Lett. 106, 135501 (2011).

[56] J. H. Los, M. I. Katsnelson, O. V. Yazyev, K. V. Zakharchenko, and A. Fasolino, Phys. Rev. B 80, 121405 (2009).

[57] J. M. H. Kroes, A. Fasolino, and M. I. Katsnelson, Phys. Chem. Chem. Phys. 18, 19359 (2016).

[58] S. K. Singh, M. Neek-Amal, S. Costamagna, and F. M. Peeters, Phys. Rev. B 87, 184106 (2013).

[59] A. Fasolino, J. H. Los, and M. I. Katsnelson, Nat. Mater. 6, 858 (2007).

[60] R. Nicklow, N. Wakabayashi, and H. G. Smith, Phys. Rev. B 5, 4951 (1972).
[61] After peer review several modifications were made in the parametrization of $\mathrm{BN}-\mathrm{ExTeP}$ to improve the description of pristine $h$-BN and $c$-BN and their melting behavior. These modifications did not affect the quality of the description of defective structures. In view of the considerable computational effort for the large amount of simulations performed for Sec. V, it was not possible to repeat them all. Therefore we repeated only part of the simulations with the final parametrization given in this paper and verified that the results are not affected.

[62] J. H. Los, K. V. Zakharchenko, M. I. Katsnelson, and A. Fasolino, Phys. Rev. B 91, 045415 (2015).

[63] J. M. Carlsson, L. M. Ghiringhelli, and A. Fasolino, Phys. Rev. B 84, 165423 (2011).

[64] G. López-Polín, C. Gómez-Navarro, V. Parente, F. Guinea, M. I. Katsnelson, F. Pérez-Murano, and J. Gómez-Herrero, Nat. Phys. 11, 26 (2015).

[65] J. H. Los, A. Fasolino, and M. I. Katsnelson, Phys. Rev. Lett. 116, 015901 (2016). 